\title{
Surgical Management of a Cervical Synovial Cyst by Minimally Invasive Surgery: Case Report with a Technical Note
}

\author{
Nanjundappa S Harshavardhana ${ }^{1}$, Joshua J Hohertz ${ }^{2}$ and James D Schwender ${ }^{2 *}$ \\ ${ }^{1}$ Dumfries and Galloway Royal Infirmary, Dumfries-Scotland, United Kingdom \\ ${ }^{2}$ Twin Cities Spine Center, Minneapolis-MN, USA
}

*Corresponding author: JD Schwender, 913E26th St\#600, Piper Building, Minneapolis, MN 55404, United States of America.

Received Date: February 01, 2021

Email ID: JDSchwender@TCSPINE.com

Published Date: March 05, 2021

\begin{abstract}
Background: Synovial cysts are most common in lumbar spine and are usually associated with degenerate facet joints. They are rare in cervical spine and less than 50 symptomatic cysts are reported in literature that were all treated by conventional open decompression either with or without fusion. Our objective was to report the surgical management of symptomatic cervical synovial cyst causing radiculo-myelopathic symptoms in an elderly gentleman treated by minimally invasive surgery.

Case Presentation: A seventy-nine-year-old gentleman presenting with three months history of progressively worsening right arm pain with myelopathic gait secondary to compression at C4-C5 caused by a synovial cyst that was treated by excision, decompression and unilateral lateral mass fixation by minimally invasive surgical approach. There was improvement in his gait and complete resolution of radiculopathy at six months postoperative follow-up visit. At five years post-op, he has remained symptom-free.

Conclusion: Excision of symptomatic CSCs can be safely performed by minimally invasive surgery technique as a day case procedure and was associated with complete resolution of neurological symptoms.
\end{abstract}

Keywords: Synovial cyst; Cervical spine; Lateral mass fixation; Minimally invasive surgery; Radiculopathy; Myelopathy

\section{Introduction}

The cervical spine is an uncommon site for synovial cysts as $>95 \%$ of them are seen in lumbar spine [1]. Cervical synovial cysts (CSC) are associated with advanced age and more common in men than women (M:F ratio of 2:1) [2]. They are most commonly solitary, unilateral and seen in both osteoarthritis (OA) and Rheumatoid arthritis (RA). Cranio-vertebral cysts are more commonly seen in RA and sub-axial CSC are most seen at C7-T1 level [3,4]. At least one case of bilateral CSC causing quadriparesis, multiple CSC affecting at least two levels (i.e.C3-C4\&C4-C5) and a CSC causing Brown-Sequard syndrome has also been reported [5-7]. Associated degenerative disc disease or spondylolisthesis is commonly seen to a varying degree in patients with CSC.

The natural history of CSC is poorly understood as they are asymptomatic and detected incidentally. However, some CSCs may be a source of compression causing radicular and myelopathic symptoms. Less than 50 such symptomatic CSCs are reported in English literature. Surgical excision with decompression with / without fusion is the mainstay of treatment for symptomatic CSC [8]. The indications for fusion of the cervical spine are debatable though it is reported to be associated with reduced recurrence 
in the lumbar spine. Very little is known regarding the role of minimally invasive surgery (MIS) or surgical excision of CSC by MIS technique in English literature.

\section{Case Report}

A seventy-nine-year-old retired right hand dominant gentleman presented to the senior author's office with a three-month history of progressively worsening right arm pain with gait disturbances and axial neck pain. He denied any history of fall, trauma or whiplash in the recent past. The pain was worse in the mornings on waking-up which marginally got better as the day progressed. He reported difficulty in bending his elbow to sip coffee and denied any difficulty or weakness in hand function/activities. His chief worry was progressive unsteadiness in feet with a fear of falling that compelled him to stay indoors relying on his son to do shopping for him. He also reported axial neck pain which was gradually getting worse despite analgesia and chiropractor treatment over the past two months. He denied having any formal physical therapy or interventional epidural injections for neck / arm pain. He also denied any bladder or bowel symptoms. The neck pain: arm pain ratio was $60: 40$ and NDI were $22 \%$ at the time of first consult.

His medical history included hypertension, atrial fibrillation, bilateral total hip arthroplasties, previous cholecystectomy and appendectomy. His most recent surgery was a knee arthroscopy four years earlier. He was a teetotaller and was otherwise in good health.

On clinical examination, he had some midline tenderness in the lower cervical spine with global restriction in neck range of motion (ROM). Motor strength testing revealed weakness in right shoulder external rotation and elbow flexion (Medical research council [MRC] grade 4/5 motor strength) with depressed Biceps jerk. He had right arm radicular pain with paraesthesia in C5 nerve root distribution. Spurling's and Rhomberg's signs was positive and was unable to perform tandem walking. Hoffman's sign was positive bilaterally and no clonus was elicited.

A working diagnosis of cervical spondylotic myelopathy (CSM) was made and MRI revealed some spondylotic /degenerative changes and without any vertebral erosion or destruction. The scans also revealed a well-defined C4-C5 cystic mass arising from the posterior elements in proximity to the facet joint on the right side dorsal to the $\mathrm{C} 4$ vertebral body and ventral to the ligamentum flavum encroaching on to the spinal canal causing abutment / compression of the exiting right $\mathrm{C} 5$ nerve root with displacement of the spinal cord / thecal sac to the left (Figures 1a and 1b).

A working diagnosis of benign juxta-facetal cyst was made after haematological work-up by ruling out a lymphoma and other neurogenic tumours. He was offered a surgical excision of cyst with decompression of the right C5 nerve root / spinal cord to arrest the progression of neurological symptoms / deterioration in function to which he consented and underwent the surgery as described below. The intraoperative findings and histology were consistent with a CSC and fluoroscopy image is shown in (Figure 2). At six weeks post-surgery, he had complete resolution of radiculopathy and myelopathic symptoms with improvement in NDI from $22 \%$ to $4 \%$. Check x-rays at final follow-up are depicted in (Figures 3a and $3 b)$. He continued to remain symptom-free with at final follow-up of five years and did not have any recurrence of the excised CSC.
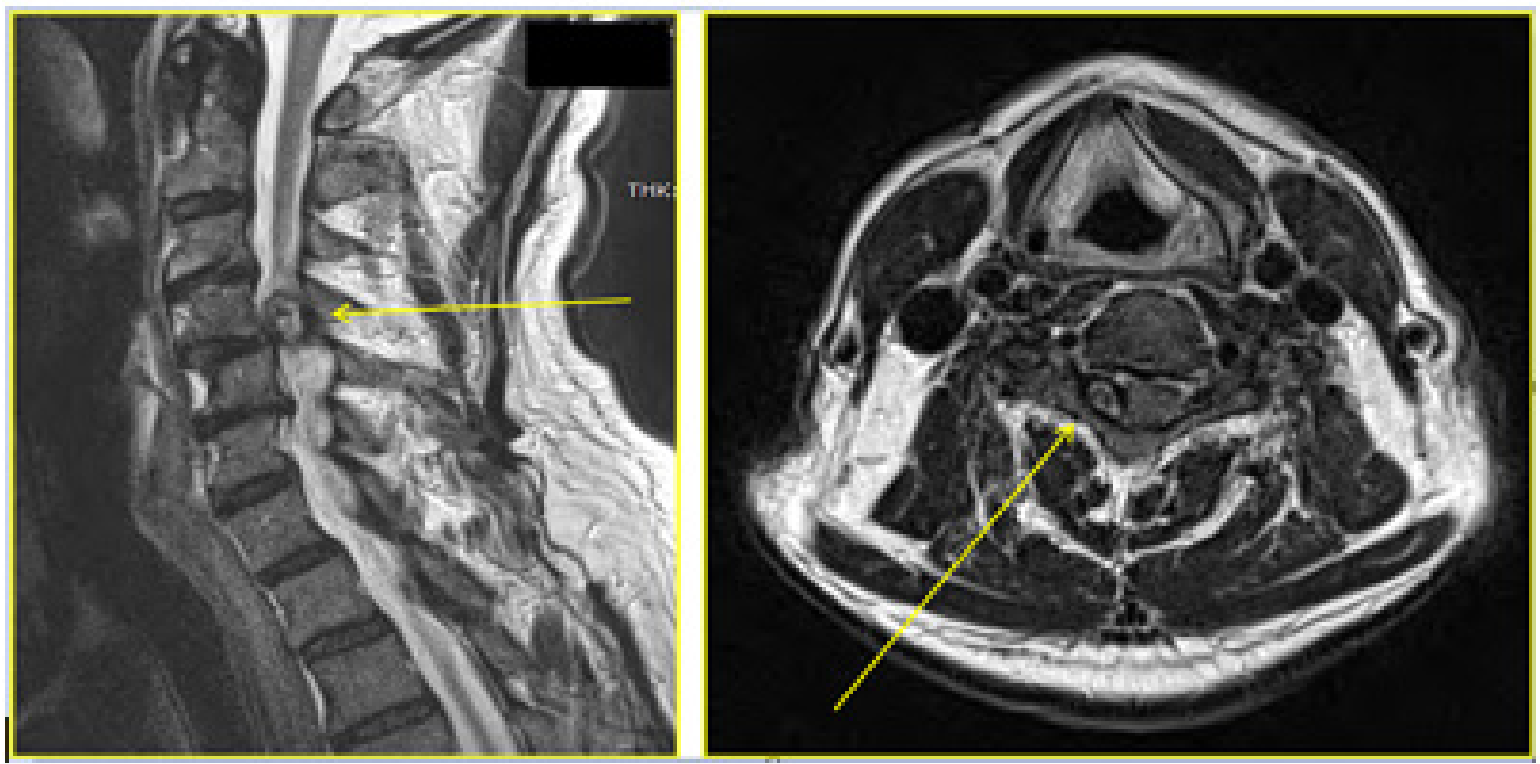

Figures 1a and 1b: Preoperative sagittal and axial T2 images of the C4-C5 synovial cyst encroaching onto the spinal canal causing compression of thecal sac. 


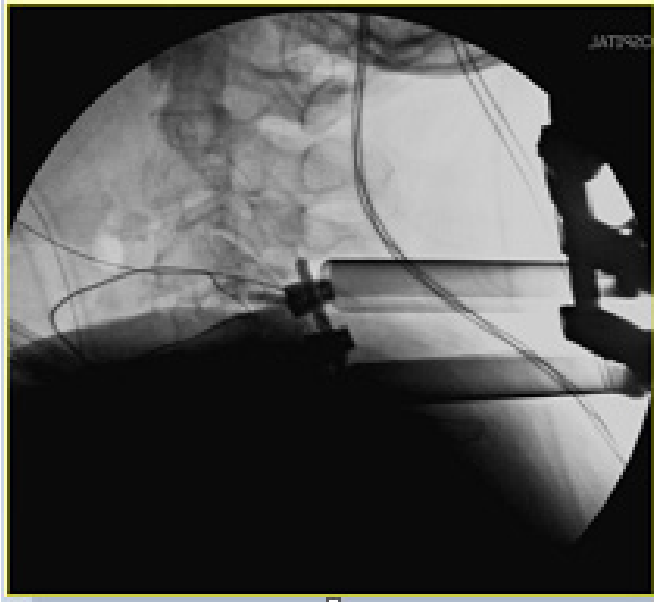

Figures 2: Intraoperative fluoroscopy image showing MAST quadrant tubular retractors with lateral mass fixation of C4 \& C5.

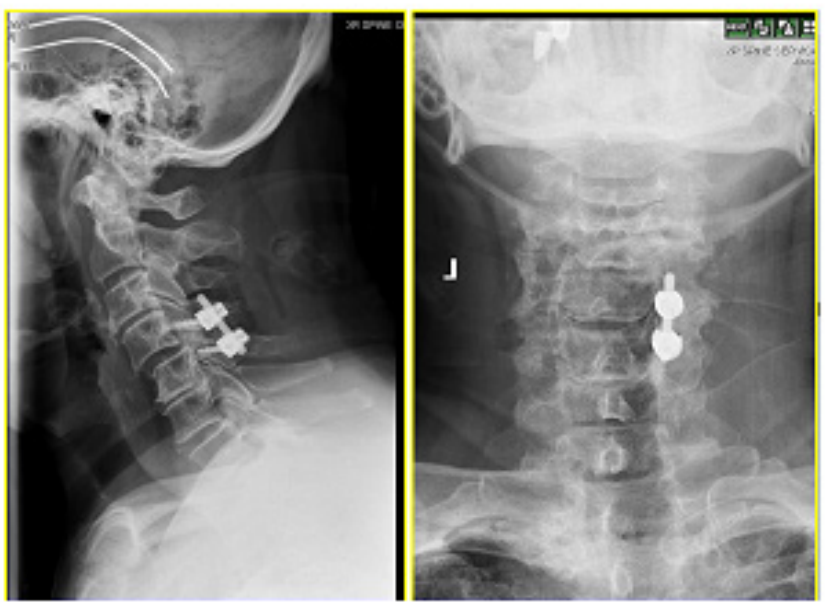

Figure 3a and 3b: Final follow-up lateral \& PA radiographs showing the unilateral lateral mass instrumentation.

\section{Surgical Technique}

The patient was administered general anaesthesia with endotracheal intubation and a Mayfield infinity skull clamp (Integra Life sciences Corporation, Cincinnati - OH; USA) was applied. He was positioned prone onto a Pro-Axis table (Mizuhosi Orthoaedics systems Inc., Union city, CA - USA) with neck in slight flexion and reverse Trendelenburg position to minimize bleeding from venous engorgement. Adequate padding of all pressure points and eyeballs were done with due care to avoid hyperabduction of upper extremities and traction induced injury to the brachial plexus [9]. A C-Arm image intensifier was used to identify the level of interest (i.e.C4-C5, the level of CSC) and a K-wire was inserted a segment below the level of interest (i.e. at C5-C6 level) 10-12mm lateral to the midline and angulated cephalad parallel to the orientation of the facet joint under fluoroscopy. The K-wire trajectory was accepted as being satisfactory with its tip resting on postero-lateral structures of C4-C5 facet joint.
A $25 \mathrm{~mm}$ longitudinal skin incisional marking was made centered over the K-wire and infiltration with $2 \mathrm{mls}$ of $0.25 \%$ bupivacaine diluted in $8 \mathrm{mls}$ of $0.9 \%$ saline having 1:1000 epinephrine (total $10 \mathrm{mls}$ ) was performed. The skin incision was made after waiting for a couple of minutes and minimal access spinal technologies (MAST) quadrant tubular retractor system rods (Medtronic Sofamor Danek, Memphis - TN; USA) were rail-roaded over the K-wire serially enlarging the soft tissues circumferentially to visualize the area of pathology. The tubular rods were removed after docking the MAST tubular retractors centered over the right C4-C5 facet joint and retractors adjusted to clearly visualize the area of interest / pathology. A monopolar cautery was used to expose the lamina, lateral mass and facet joint. Pituitary ronguer was used to clear the soft tissues / muscles and a bipolar cautery used to secure haemostasis. Care was taken to visualize the entire medio-lateral extent of the lateral mass. Hemilaminotomy of $\mathrm{C} 4$ was performed with satisfactory decompression of the cervical cord with en- 
masse removal of the cyst material. Meticulous dissection was performed to separate the ventral cyst wall from the dura without causing a durotomy / tear. The pulsatile dura following removal of compressing/ pathological cyst material signified the completion of satisfactory decompression which warranted removal of part of the articular process of facet joint. The synovium of facet joint was removed with a curette and remnant cartilage covering facet joint was scored off to expose the underlying cancellous bony bed. Allograft granules admixed with bone marrow aspirate from the iliac crest was then packed to facilitate facetal arthrodesis.

A $2 \mathrm{~mm}$ high-speed burr was used to create entry point for lateral mass screw by Roy-Camille technique at the centre of lateral masses of C4 \& C5 [10]. A $2.5 \mathrm{~mm}$ drill bit with a $12 \mathrm{~mm}$ stopping length was used to create a pilot hole with its trajectory directed 15 20 degrees cephalad under fluoroscopy guidance. This orientation avoided entering the facet joint or exiting nerve root inferiorly or the vertebral artery located in the midline of the lateral mass. The trajectory track depth was measured, tapped and the four walls were felt with a ball-tipped probe to confirm the thus created channel was intra-osseous in its entirety. Two $3.5 \mathrm{~mm} \mathrm{X} 12 \mathrm{~mm}$ polyaxial lateral mass screws were then inserted into the prepared channels. A $3.5 \mathrm{~mm}$ titanium rod of appropriate length was then laid on the top-opening polyaxial screw heads and set-screws were inserted / tightened using torque tensioner (Figure 2). The dorsal surfaces of lateral masses were then decorticated and residual bone graft were laid lateral to the rod to facilitate sound arthrodesis. The surgical scar was closed with 2-0 vicryl, 3-0 monocryl, dermabond with steri-strips to the skin and patient was discharged home the same day.

\section{Discussion}

Cysts in cervical spine could be either true synovial cysts or false ganglion cysts based on histopathological evaluation [11]. The true CSC have a synovial cyst wall lining and a clear / xanthochromic fluid encased within it [12]. The false ganglion cysts lack a mesothelial wall lining and may have a gelatinous fluid2. Nevertheless, their clinical presentation, treatment and prognosis is identical and usually discussed as a single entity. These cysts are most commonly associated with degenerated and segmentally unstable spine and are usually seen in elderly patients. They could be either cranio-vertebral (i.e.O-C2) or sub-axial (C3C7) in location. Symptomatic cranio-vertebral CSC most commonly present with myelopathic features unlike the sub-axial ones that can manifest with either myelopathy and / or radiculopathy. It was hypothesised that the degeneration coupled with segmental instability caused synovial hypertrophy with protrusion through the small joint capsular defects into the epidural compartment forming a para-articular cavity. The mechanically stresses were also found to initiate an inflammatory cascade with upregulation of Interleukin-1, Interleukin-6, Platelet derived growth factor (PDGF), Epidermal growth factor (EGF) and other cytokines that may cause neovascularisation, hyperplasia with exudation of synovial fluid $[13,14]$.

In a meta-analysis of 101 patients with CSC, Bydon M et al reported their incidence to be $4.9 \%$ in Rheumatoid arthritis (RA), $7.9 \%$ in congenital cervical fusions, $10.9 \%$ of cervical trauma, $18.8 \%$ with co-existent spondylolisthesis and $26.7 \%$ with degenerated discs [15]. In the largest reported case series of 35 patients with sub-axial CSC, Lyons $\mathrm{MK}$ et al found a high incidence (almost $50 \%$ ) at C7-T1 level and hypothesised that a transition zone with translational facet joint that was subjected to unique biomechanical forces / vectors to be the probable cause that predisposed the C7T1 facet joints to be the preferred site [4].

Management of CSC has traditionally been observation and regular follow-up for asymptomatic ones with interventional management for symptomatic cysts. Spontaneous resolution of CSCs is not uncommon [14]. Though aspiration of cyst wall under CT / fluoroscopic guidance has been reported [16], symptomatic CSCs are best treated surgically $[2,4,15]$. There is no consensus on standard of care for symptomatic CSCs and surgical strategies have varied from instrumented fusion without cyst excision to cyst excision plus decompression without fusion and decompression with fusion (instrumented or otherwise). Uninstrumented fusion was associated with higher risk of recurrence $1.8 \%$ ) in comparison to instrumented fusion for lumbar synovial cysts and is unknown for CSC $[8,15]$. We were unable to find any comparative studies (Level of Evidence II or III) that had evaluated the role of decompression alone vs. decompression+fusion or instrumented vs. un-instrumented fusion or unilateral vs. bilateral instrumentation for CSCs in English literature.

With advances in surgical techniques and emerging role of minimally invasive cervical spine surgeries that is increasingly performed in contemporary era, we were unable to find any published case report that has reported successful management of CSC by MIS. MIS has distinct advantages over open surgeries in minimizing the soft tissue/muscle dissection maintaining the integrity of dynamic posterior tension band with faster recovery and reduced incidence of axial neck pain $[17,18]$. Though MIS has hitherto been employed in treating cervical spine trauma, pseudoarthrosis and for stabilization following anterior metastatic tumour removal, its potential role could be extended to successfully treat other pathologies too (i.e. benign cysts, locally aggressive tumours etc...). Larger case series with longer follow-up (LoE IV) and prospective comparative studies evaluating outcomes of MIS against open access excision (LoE II) is desired to establish the superiority of one technique over the other and advocate routine use of MIS in operative management of CSCs.

\section{Conclusion}

An index case of symptomatic cervical Synovial Cyst (CSC) successfully managed by excision and unilateral lateral mass 
stabilization using minimally invasive system (MIS) with a minimum follow-up of five years is being presented. There was no recurrence of either the cyst or neurological symptoms (myelopathy or radiculopathy) at final follow-up of five years.

\section{Acknowledgement}

None.

\section{Conflict of Interest}

All authors do not have any CoI to disclose and no funding from any source was received by any of us for this research.

\section{References}

1. Christophis P, Asamoto S, Kuchelmeister K, Walter Schachenmayr (2007) Juxtafacet cysts: a misleading name for cystic formations of mobile spine (CYFMOS). Eur Spine J 16(9): 1499-505.

2. Shima Y, Rothman SL, Yasura K, Shinobu Takahashi (2002) Degenerative intraspinal cyst of the cervical spine: case report and literature review. Spine (Phila 1976) 27(1): E18-22.

3. Morio Y, Yoshika T, Nagashima H (2003) Intraspinal synovial cyst communicating with the C1-C2 facet joints and subarachnoid space associated with rheumatoid atlantoaxial instability. Spine (Phila 1976); 28(23): E492-495.

4. Lyons MK, Birch BD, Krauss WE, Naresh P Patel, Eric W Nottmeier, et al (2011) Subaxial cervical synovial cysts: report of 35 histologically confirmed surgically treated cases and review of the literature. Spine (Phila 1976) 36(20): E1285-1289.

5. Kotilainen E, Marttila RJ (1997) Paraparesis caused by a bilateral cervical synovial cyst. Acta Neurol Scand 96(1): 59-61.

6. Nijensohn E, Russell EJ, Milan M, T Brown (1990) Calcified synovial cyst of the cervical spine: CT and MR evaluation. J Comput Assist Tomogr 14(3): 473-476.

7. Kim SW, Ju CI, Kim HS, Yun Sung Kim (2014) Brown-sequard syndrome caused by a cervical synovial cyst. J Korean Neurosurg Soc 55(4): 215217.
8. Bydon A, Xu R, Parker SL, Mohamad Bydon, Ziya L Gokaslan, et al (2010) Recurrent back and leg pain and cyst reformation after surgical resection of spinal synovial cysts: systematic review of reported postoperative outcomes. Spine J 10(9): 820-826.

9. Schwartz DM, Drummond DS, Hahn M, J P Dormans (2000) Prevention of positional brachial plexopathy during surgical correction of scoliosis. J Spinal Disord 13(2): 178-182.

10. Roy-Camille R, Saillant, Mazel C (1989) Internal fixation of the unstable cervical spine by a posterior osteosynthesis with plates and screws. The cervical spine 2nd ed, Philadelphia, JB Lippincott(eds) pp. 390-403.

11. Bisson EF, Sauri-Barazza JC, Niazi T, Meic H Schmidt (2013) Synovial cysts of the cervicothoracic junction causing myelopathy: report of 3 cases and review of the literature. Neurosurg Focus 35(1): E3.

12. Kao CC, Winkler SS, Turner JH (1974) Synovial cyst of spinal facet. Case report. J Neurosurg 41(3): 372-376.

13. McGuigan C, Stevens J, Gabriel CM (2005) A synovial cyst in the cervical spine causing acute spinal cord compression. Neurology 65(8): 1293.

14. Colen CB, Rengachary S (2006) Spontaneous resolution of a cervical synovial cyst. Case illustration. J Neurosurg Spine 4(2): 186.

15. Bydon M, Lin JA, de la Garza-Ramos, Daniel M Sciubba, Jean Paul Wolinsky, et al (2014) The role of spinal fusion in the treatment of cervical synovial cysts: a series of 17 cases and meta-analysis. J Neurosurg Spine 21(6): 919-928.

16. Kostanian VJ, Mathews MS (2007) CT Guided Aspiration of a Cervical Synovial Cyst. Case Report and Technical note. Interv Neuroradiol 13(3): 295-298.

17. Wang MY, Levi AD (2006) Minimally invasive lateral mass screw fixation in the cervical spine: initial clinical experience with long-term follow-up. Neurosurgery 58(5): 907-912.

18. Oppenheimer JH, DeCastro I, McDonnell DE (2009) Minimally invasive spine technology and minimally invasive spine surgery: a historical review. Neurosurg Focus 27(3): E9. 